\title{
Pre-clinical assessment of chimeric antigen receptor $t$ cell therapy targeting CD19+ B cell malignancy
}

\author{
Sheng-I Hu${ }^{1 \#}$, Ming-Chin Ko ${ }^{2 \#}$, Yi-Han Dai ${ }^{2 \#}$, Hsin-An Lin ${ }^{3}$, Lih-Chyang Chen ${ }^{4}$, Kuo-Yang Huang ${ }^{5}$, \\ Te-Ling Pang ${ }^{2}$, Cheng-Yi Kuo ${ }^{2,6}$, Hsin-Chung Lin $^{7}$
}

${ }^{1}$ Division of Colorectal Surgery, Department of Surgery, Tri-Service General Hospital, National Defense Medical Center, Taipei City, Taiwan; ${ }^{2}$ Uwell Biopharma Inc., New Taipei City, Taiwan; ${ }^{3}$ Division of Infection, Department of Medicine, Tri-Service General Hospital SongShan Branch, National Defense Medical Center, Taipei City, Taiwan; ${ }^{4}$ Department of Medicine, Mackay Medical College, New Taipei City, Taiwan; ${ }^{5}$ Graduate Institute of Pathology and Parasitology, National Defense Medical Center, Taipei City, Taiwan; ${ }^{6}$ Department and Graduate Institute of Biology and Anatomy, National Defense Medical Center, Taipei City, Taiwan; ${ }^{7}$ Division of Clinical Pathology, Department of Pathology, Tri-Service General Hospital, National Defense Medical Center, Taipei City, Taiwan

Contributions: (I) Conception and design: SI Hu, MC Ko, YH Dai, TL Pang, CY Kuo, HC Lin; (II) Administrative support: HA Lin, LC Chen, KY Huang; (III) Provision of study materials or patients: All authors; (IV) Collection and assembly of data: MC Ko, YH Dai, TL Pang, CY Kuo; (V) Data analysis and interpretation: MC Ko, YH Dai, TL Pang, CY Kuo; (VI) Manuscript writing: All authors; (VII) Final approval of manuscript: All authors.

${ }^{\#}$ These authors contributed equally to the work.

Correspondence to: Cheng-Yi Kuo, PhD. Rm. 10, 6F., No. 97, Sec. 1, Xintai 5th Rd., Xizhi Dist., New Taipei City 22175, Taiwan.

Email: Jerry.kuo@Uwell.com.tw; Hsin-Chung Lin. No. 325, Section 2, Cheng-Kung Road, Neihu District, Taipei City 11490, Taiwan.

Email: hsinchunglin@gmail.com; Te-Ling Pang, PhD. Rm. 10, 6F., No. 97, Sec. 1, Xintai 5th Rd., Xizhi Dist., New Taipei City 22175 , Taiwan.

Email: celine.pang@uwell.com.tw.

Background: Autologous chimeric antigen receptor (CAR) $\mathrm{T}$ cell therapy is a promising therapeutic strategy for treating hematologic malignancies. A spectrum of serious complications caused by CAR-T cells has caught great attention. We developed a novel CAR against CD19 namely UWC19, consisting antiCD19 single-chain variable fragment (scFv) hinged with 4-1BB and CD3z signaling domains. In this study, preclinical assessments of UWC19 were conducted to evaluate the safety and efficacy in vitro and in vivo.

Methods: To evaluate the binding activity of UWC19 cells to CD19, we measured the saturation degree of CAR with human CD19 molecules using flow cytometry in vitro. The antitumor efficacy of UWC19 cells was determined by in vitro cytotoxicity assay against CD19 positive cells and in vivo using a xenograft mouse model. Cross tissue reactivity of UWC19 cells was examined by co-culturing with cell lines from difference human tissues. Tumorigenicity was determined by subcutaneously injecting UWC19 in immunodeficient mice. Persistence was analyzed using quantitative PCR.

Results: We showed that UWC19 CAR T cells exerted highly specific binding affinity and cytotoxicity against CD19+ cells in vitro. In vivo, UWC19 CAR T cells are able to fully control disease progression in a Raji-xenografted immunodeficient mouse model. UWC19 exerted no obvious effects on the mean body mass and graft versus host disease were observed in surviving mice. We showed that UWC19 cells specifically recognized and eliminated CD19 positive cells, whereas CD19 negative cells were much less affected. No tumorigenicity of UWC19 in immunodeficient mice was observed.

Conclusions: UWC19 treatment effectively eliminated CD19 positive tumor cells with favorable toxicity profile. The findings suggest encouraging clinical prospects for its use in patients with CD19 positive B cell malignancies. Our study presented an alternative evaluation strategy for CAR-T cell products.

Keywords: Chimeric antigen receptor (CAR); safety evaluation; tumorigenicity; immunodeficient mice; CD19; UWC19

Submitted Dec 19, 2019. Accepted for publication Feb 07, 2020.

doi: $10.21037 /$ atm.2020.02.148

View this article at: http://dx.doi.org/10.21037/atm.2020.02.148 


\section{Introduction}

Chimeric antigen receptors (CARs) are synthetic proteins composed of antigen-specific single-chain variable fragment $(\mathrm{scFv})$ and intracellular activating signaling domains, which is designed to re-direct $\mathrm{T}$ cells to target cells expressing specific cell-surface antigens. T cells armed with CAR specifically recognizing their target antigen through the $\mathrm{scFv}$ binding domain, leading to $\mathrm{T}$ cell activation in a nonmajor histocompatibility complex (MHC) manner. The use of CAR-T cells has been demonstrated to be a promising treatment against hematological malignancies with successes in the autologous setting $(1,2)$. For B cell malignancies, anti-CD19 CAR-T cells have been demonstrated to induce complete remission in patients with refractory acute lymphoblastic leukemia $(3,4)$. A recent trial has reported that up to $90 \%$ of patients with relapsed or refractory ALL achieved complete remission with a cell persistence of 6 months (5). In contrast, it has been shown that CAR-T cells exerted less graft-versus-tumor activities in patients with non-Hodgkin lymphoma and chronic lymphocytic leukemia $(6,7)$. Despite the promising results in clinical setting, there are fields in which unique toxicities such as cytokine release syndrome and short persistence after re-infusion pose new challenges. In addition, tumorigenicity and off-target toxicity remain as great concern for its clinical use.

CD19-targeted second-generation CARs derived from FMC63 scFv have been demonstrated for their therapeutic uses in clinical trials. In addition, clinical trials targeting CD19 have utilized other second-generation CARs derived SJ25C1-mAb in patients with relapsed or refractory B-cell leukemia $(8,9)$. Given clinical experiences, antitumor activities have been critically depended on the design of CARs. In addition, CARs derived from non-human mAb have been shown to elicit cellular and humoral immune responses leading to unfavorable persistence after infusion (10). In this study, we described a construction of CD19-specific CARs and examined its anti-CD19+ malignancy activities with emphases on cytokine release, residual leukemia cells, cytolytic activity and xenograft mice study. Moreover, we evaluated the tumorigenecity and cross tissue reactivity of the construct.

\section{Methods}

\section{Tumor cell lines}

The 293T cells and human leukemia cell lines SUP-T1 and Raji cells were purchased from the American Type Culture
Collection (ATCC; Rockville, MD). The human leukemia cell line K562 was purchased from the Bioresource Collection and Research Center (BCRC; Hsinchu, Taiwan). The Raji, SUP-T1 and K562 cells were maintained in RPMI-1640 (Invitrogen, Carlsbad, CA) and the 293 T cells was maintained in DMEM (HyClone; GE Healthcare, South Logan, UT). Media were supplemented with $10 \%$ fetal bovine serum (FBS; Gibco, Carlsbad, CA) and $100 \mathrm{mg} / \mathrm{mL}$ penicillin/streptomycin (Gibco, Carlsbad, CA). For the visualization of injected tumor cells in immunodeficient mice, the Raji cells were transduced with a Lentivirus-EF1alpha-MCS- IRES-Luciferase plasmid (Addgene, Cambridge, MA) and the single Raji-Luc cell with the highest expression of luciferase was sorted with a FACSAria cell sorter (BD Biosciences, San Jose, CA). Expression of CD19 on B-cell ALL cell lines was detected using an anti-human CD19 antibody conjugated to phycoerythrin (PE; BD Biosciences, San Jose, CA) and was visualized using a FACSCalibur flow cytometry (BD Biosciences, San Jose, CA).

\section{Transduction of human T cells}

Peripheral blood samples were obtained from discarded anonymized by-products of platelet donations from healthy adult donors. Mononuclear cells collected from the samples by centrifugation on a Lymphoprep density step (Nycomed, Oslo, Norway) were washed twice using medium. Human T cells were purified by negative selection with a mixture of CD14, CD16, CD19, CD36, CD56, CD123, and CD235a antibodies and magnetic separation (Pan T Cell Isolation Kit II; Miltenyi Biotec). Purified T cells were transduced with thawed lentiviruses to generate CAR-T cells possessing CD19 binding domains. The resulting $\mathrm{T}$ cells were stained with Biotin-SP-conjugated AffiniPure Goat anti-mouse IgG,F(ab')2 fragment specific antibody (Jackson ImmunoResearch, West Grove, PA) followed by PEconjugated streptavidin (Jackson ImmunoResearch, West Grove, PA) to determine the percentage of CAR-positive $\mathrm{T}$ cells.

\section{In vitro cytotoxic assay}

CD19 positive Raji cells were used as target cells, whereas CD19 negative K562 cells served as control cells. The antiCD19 CAR-T cells and target cells were co-cultured in 96well plates with indicated effector to target $(\mathrm{E}: \mathrm{T})$ ratios. After 24 hours, cells were harvested, washed and stained 
Table 1 Cell lines used in this study

\begin{tabular}{lcl}
\hline Organ & Cell line & Cell type \\
\hline Bone marrow & K562 & Human chronic myeloid leukemia cells \\
Bone marrow & K562/CD19+ & Human chronic myeloid leukemia cells \\
Lung & A549 & Human alveolar basal epithelial cells \\
Lymphoblast & Raji & Human B cell lymphoma cells \\
Kidney & HK2 & Human kidney proximal tubules epithelial cells \\
Colon & HT-29 & Human colon epithelial cells \\
Kidney & $768-O$ & Human renal carcinoma cells \\
Liver & HepG2 & Human hepatic epithelial cells \\
Breast & MDA-MB-231 & Human mammary gland epithelial cells \\
\hline
\end{tabular}

with PE-conjugated mouse anti-human CD19 antibody (BD Biosciences, San Jose, CA). The percentage of CD19 positive cells representing the level of residual leukemia cells was analyzed by FACSCalibur flow cytometry. The supernatants were harvested for the measurement of IFN- $\gamma$ production of the CAR-T cells in response to leukemia cells using ELISA kits (R\&D Systems, Inc., Minneapolis, MN). The absorbance at $450 \mathrm{~nm}$ was measured using a microplate reader (Anthos 2010, Biochrom Ltd., Cambridge, UK). Cytotoxicity assay was further analyzed to determine the specific anti-leukemic ability of CAR-T cells. The target cells were suspended in RPMI-1640 containing $10 \%$ FBS, labeled with calcein AM (BD Biosciences, San Jose, CA), and plated onto 96-well flat-bottom plates (Costar, Corning, NY). The CAR-T cells, suspended in RPMI-1640 containing $10 \%$ FBS, were then added at various $\mathrm{E}$ : $\mathrm{T}$ ratios and co-cultured with the target cells for $4 \mathrm{~h}$. Following this, the cells were stained with propidium iodide (SigmaAldrich, St. Louis, MO), and the cytotoxicity was assessed by flow cytometry on a FACSCalibur (Becton Dickinson) instrument enumerating the number of viable target cells (calcein AM-positive, propidium-iodide negative, and light scattering properties of viable cells).

\section{Xenograft model}

Raji cells expressing luciferase were injected intravenously (IV) in 6- to 8-week-old NOD.Cg-Prkdcscid Il2rgtm1Wjl/ YckNarl mice (National Laboratory Animal Center, Taiwan; $5 \times 10^{5}$ per mouse). CAR-T cells in RPMI- 1640 plus $10 \%$ FBS were IV injected in to mice at concentrations of 106, 107 and 108 cells/ml 7 days post-Raji-Luc injection.
Control group of mice received tissue culture medium instead of CAR-T cells. Raji engraftment and progression was evaluated using an IVIS Spectrum (Caliper Life Sciences, Hopkinton, MA). Mice were euthanized when bioluminescence reached $1 \times 10^{10}$ photons/second, or earlier if they showed physical signs warranting euthanasia. All animal experiments were performed in accordance with a protocol approved by the Taipei medical University Institutional Animal Care and Use Committee (IACUC) (IACUC No.: LAC-2017-0124).

Two groups of 6 animals were subcutaneously administered with UWC19 once. The animals necropsied on Day 82 for evaluation of tumorigenic potential of UWC19. In addition, 13 mice were used as the negative and positive control by injecting with DPBS, HeLa and Raji cells, respectively. Animals were treated with $1 \times 10^{6}$ and $1 \times 10^{7}$ of anti-CD19 CAR T cells. Control animals were housed in the same room, and handled in the same manner as the mice that are administered with DPBS, HeLa and Raji cells.

\section{Cross tissue reactivity assay}

Anti-CD19 CAR T cells were co-cultured with target cell lines with designated E:T ratios (1:1 and 5:1) for 4 hours (Table 1). CD19 positive Raji cells and CD19 negative K562 cells were used as positive and negative control cells respectively. Target cells were subsequently stained with PE-conjugated mouse anti-human CD19 antibody (BD Biosciences, San Jose, CA) and analyzed by FACSCanto flow cytometry. The target cells were suspended in RPMI1640 containing $10 \%$ FBS, labeled with calcein AM (BD 
Table 2 Treatment regimen for tumorigenicity study

\begin{tabular}{lc}
\hline Group & Dose (total cell injected) \\
\hline Positive control (Raji) & $1 \times 10^{7}$ \\
Positive control (HeLa) & $2 \times 10^{6}$ \\
DPBS & $\mathrm{N} / \mathrm{A}$ \\
anti-CD19 CAR-T cells & $1 \times 10^{6}$ \\
anti-CD19 CAR-T cells & $1 \times 10^{7}$ \\
\hline
\end{tabular}

CAR, chimeric antigen receptor.

Biosciences, San Jose, CA), and plated onto 96-well flatbottom plates (Costar, Corning, NY). UWC19 cells, suspended in RPMI-1640 containing 10\% FBS, were then added at various E:T ratios and co-cultured with the target cells for $4 \mathrm{~h}$. Following this, the cells were stained with propidium iodide (Sigma-Aldrich, St. Louis, MO), and the cytotoxicity was assessed by flow cytometry on a FACSCanto (Becton Dickinson) instrument enumerating the number of viable target cells (calcein AM-positive, propidium-iodide negative, and light scattering properties of viable cells).

\section{Tumorigenicity}

Animals were subcutaneously administered with $1 \times 10^{6}$ and $1 \times 10^{7}$ of anti-CD19 CAR-T cells once. The animals necropsied on Day 82 for evaluation of tumorigenic potential of anti-CD19 CAR-T cells. In addition, mice were used as the negative and positive control by injecting with DPBS, HeLa and Raji cells, respectively. Control animals were housed in the same room, and handled in the same manner as the mice that are administered with DPBS, HeLa and Raji cells (Table 2).

\section{Statistical analysis}

The values are given as mean \pm standard deviation $(\mathrm{SD})$. All results were analyzed by Duncan's multiple range test with Statistical Analysis System (SAS Institute Inc., SAS Campus Drive Cary, NC) software. A P value $<0.05$ indicated a significant difference.

\section{Results}

\section{Specific affinity and cytotoxicity of anti-CD19 CAR T cells}

The CAR-T cells expressed anti-CD19 CAR and specifically recognized FITC-labeled human CD19, whereas the binding to CD19 was absent in non-transduced T cells (Figure 1). Saturation analysis showed that binding activity of anti-CD19 CAR T cells to FITC-labeled human CD19 was saturable at a concentration of $1 \mu \mathrm{g} / \mathrm{mL}$ human CD19 (Figure 2). Anti-CD19 CAR-T cells against malignant CD19+ B cells was examined by co-culturing with RS4;11, Raji or K562 cells for 4 hours. The results showed that anti-CD19 CAR-T cells exerted CD19 specific cytotoxicity against RS4; 11 and Raji cells, whereas growth of K562 cell was slightly less inhibited (Figure 3).

\section{Inbibitory effects of anti-CD19 CAR-T cells on growth of malignant CD19+ B cell in NGS mice}

Mice treated with $1 \times 10^{7}$ of anti-CD19 CAR-T cells survived to the end of study. Survivals of mice treated with $1 \times 10^{5}$ and $1 \times 10^{6}$ of anti-CD19 CAR-T cells were less than that of group of $1 \times 10^{7}$ cells (Figure 4). All Raji-bearing mice without treatment with Welgenaleucel (UWC19) died prior to terminal sacrifice. Anti-tumor activity of CAR-T cells against CD19+ B cell lymphoma was evaluated in Rajibearing immunocompromised mice. Mice were engrafted intraperitoneally with luciferase-labeled Raji cells $\left(5 \times 10^{5}\right.$ per mouse) and subsequently injected with anti-CD19 CAR-T cells at different concentrations 6 days after tumor inoculation. Tumors aggressively developed in all mice 6 days post-inoculation. Mice treated with anti-CD19 CAR-T cells at 107 exhibited significantly lower tumor luminescence compared with that of the other groups (Figure 5).

All animal body weights were monitored prior to, throughout the study, and at sacrifice. Body weights were measured at designated time points and summarized in Table 3. The data showed that Raji lymphoma xenograft had no impact on the mean body weight over the period of study. All mice receiving anti-CD19 CAR-T cells appeared clinically normal and body weight changes were within the normal physiological range throughout the study.

\section{Persistence of anti-CD19 CAR-T cells in Raji xenograft mice}

Persistence and proliferation anti-CD19 CAR-T cells in Raji xenografted immunodeficient mice were evaluated using flow cytometry. The data showed that the total cell number of anti-CD19 CAR-T cells in the mice receiving $1 \times 10^{7}$ cells increased in 12 days after infusion, whereas 


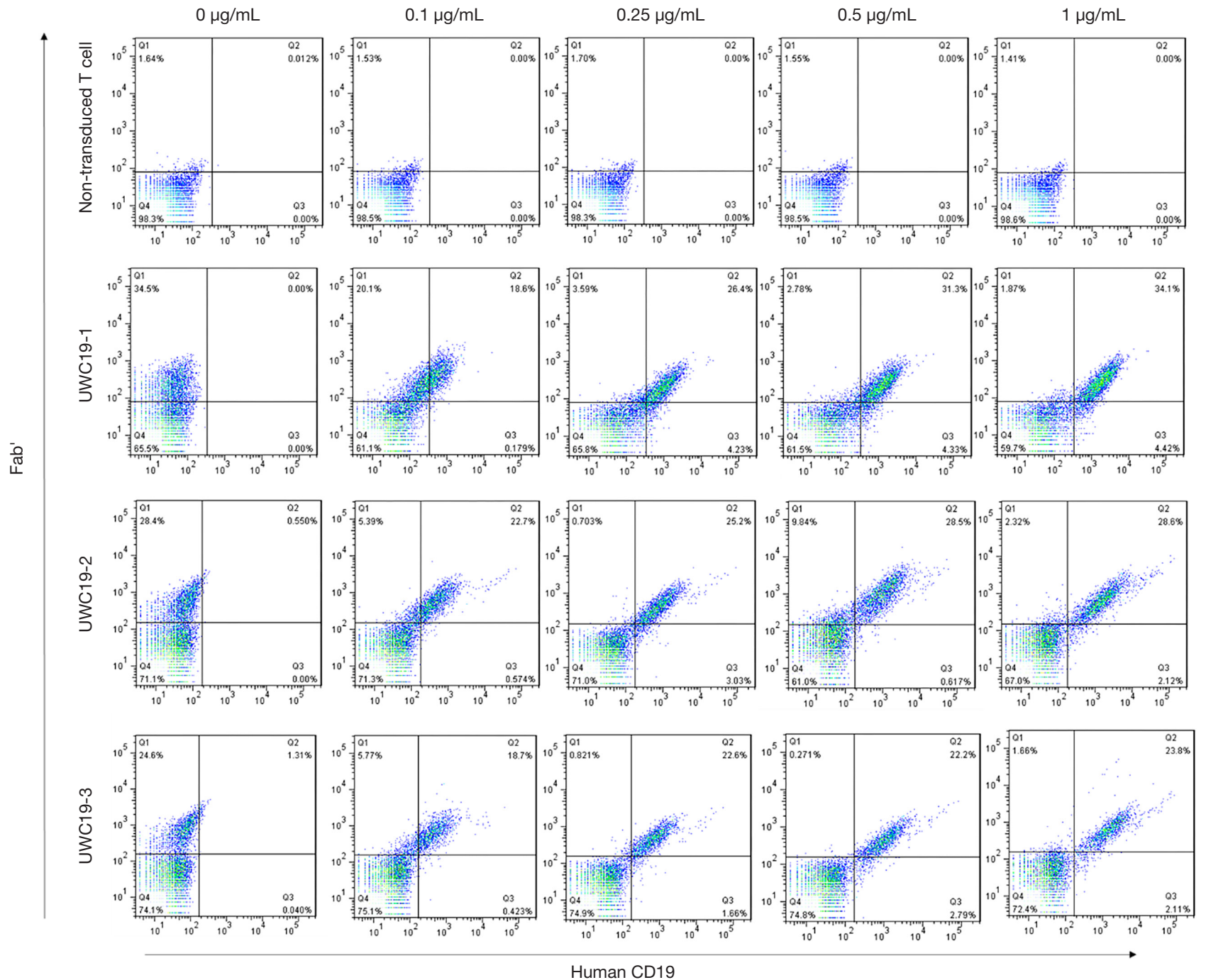

Figure 1 Binding affinity of anti-CD19 CAR T cells to human CD 19. CAR, chimeric antigen receptor.

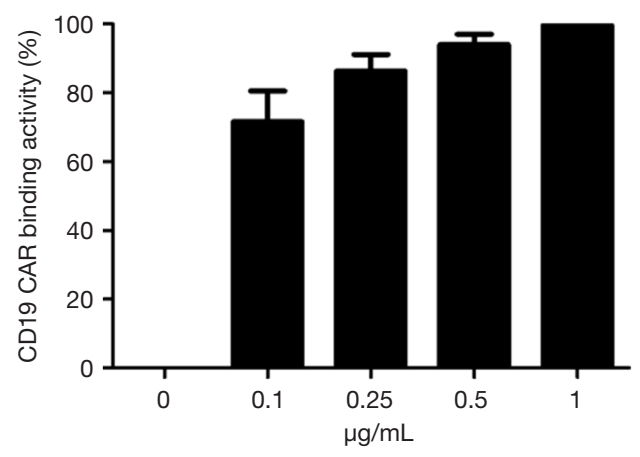

Figure 2 Saturation binding measurement. Binding of FITCconjugated human CD19 molecules to anti-CD19 CAR T cells was evaluated using flow cytometry. CAR, chimeric antigen receptor.

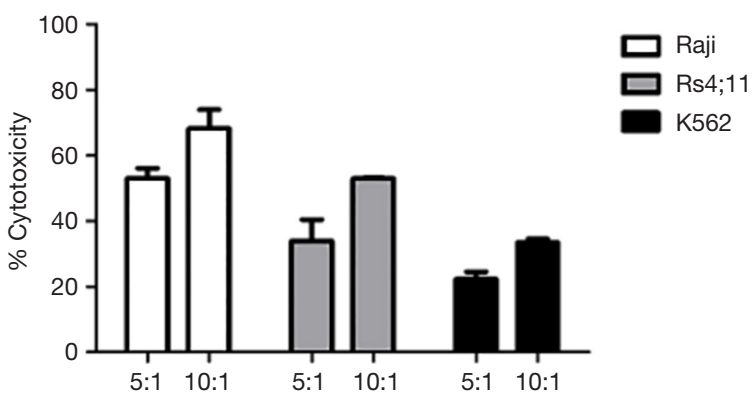

Figure 3 In vitro cytotoxicity assays of anti-CD19 CAR T cells. Percentage of residual RS4; 11, Raji and K562 cells analyzed by flow cytometry after 4 hours co-culture anti-CD19 CAR T cells at E:T ratios of 5:1 and 10:1, respectively. CAR, chimeric antigen receptor. 


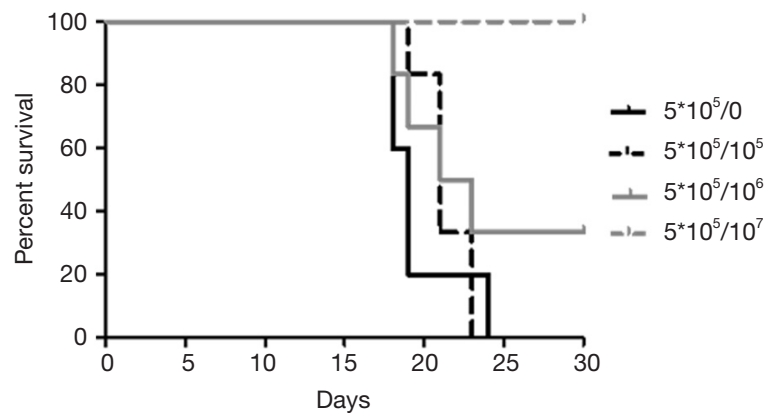

Figure 4 Kaplan-Meier survival curves for Raji-bearing mice treated with or without anti-CD19 CAR T cells in the 30-day study. CAR, chimeric antigen receptor. the other groups exhibited no proliferation of anti-CD19 CAR-T cells in the same period. Anti-CD19 CAR-T cells was detected in mice treated with $1 \times 10^{6}$ and $1 \times 10^{7}$ cells 30 days after infusion (Figure 6).

\section{In vitro cross reactivity of anti-CD19 CAR-T cells}

All target cell lines were analyzed for positivity of CD19 (Figure 7A). The results showed that all target cells exhibited significantly low CD19 expression, whereas K562/CD19+ and Raji cells had high positive rate of CD19 ( $\geq 97 \%)$. To investigate anti-CD19 CAR-T cells reactivity on the other non-target tissues, a panel of cell lines, representative of
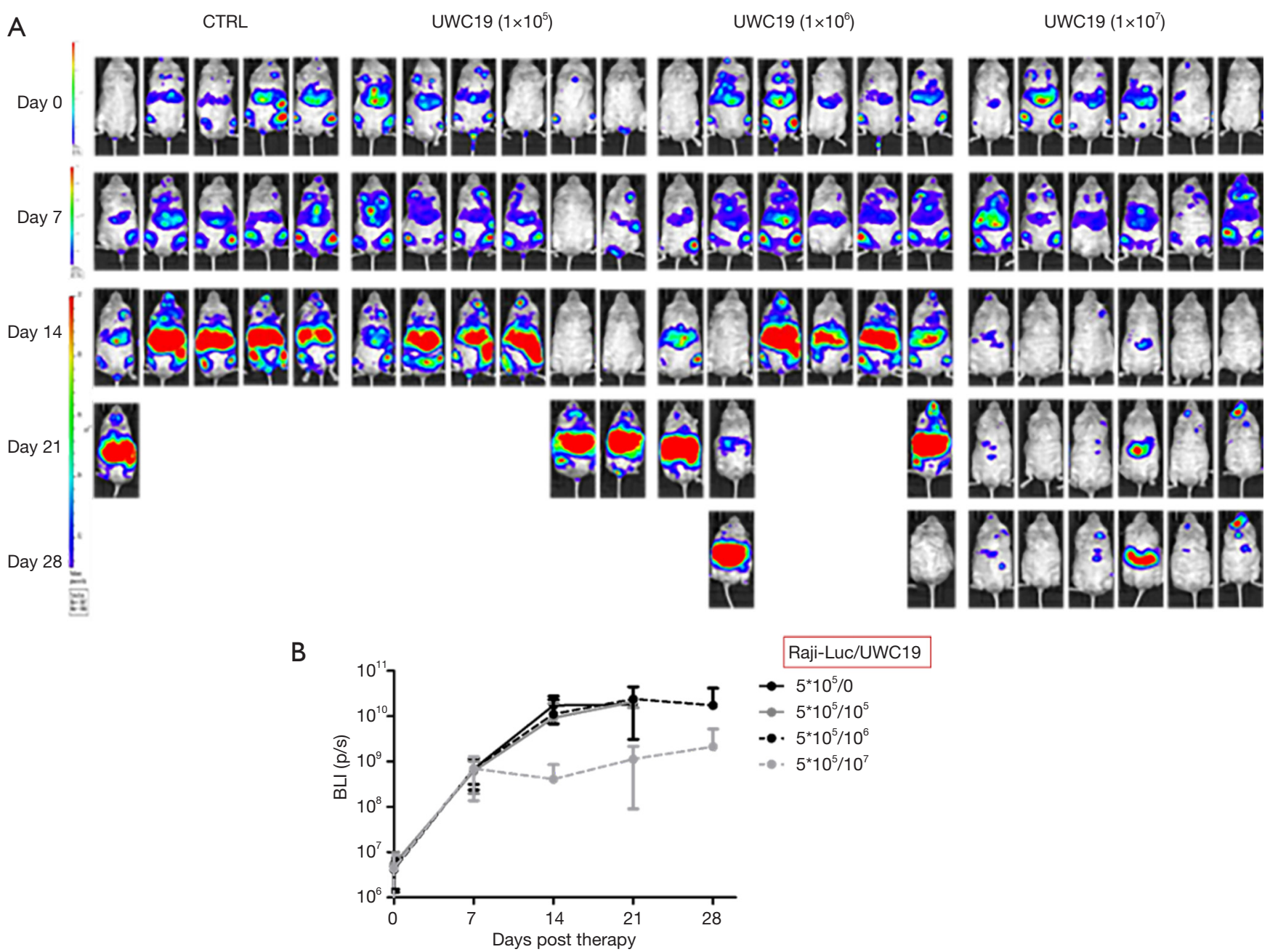

Figure 5 In vivo imaging of Raji expressing luciferase in immunodeficient mice after intravenous injection of anti-CD19 CAR T cells. (A) Mouse images of IVIS signal acquired on Day 0, 7, 14, 21 and 28; (B) quantitative analysis of IVIS signals over time. BLI, bioluminescence imaging; Luc, luciferase; CAR, chimeric antigen receptor. 
Table 3 Mean body weights of Raji-bearing immunodeficient mice receiving anti-CD19 CAR-T cells

\begin{tabular}{|c|c|c|c|c|c|c|c|}
\hline \multirow{2}{*}{ Group } & \multicolumn{7}{|c|}{ Body weight (mean \pm SD) } \\
\hline & -7 & 0 & 1 & 7 & 14 & 21 & 28 \\
\hline \multicolumn{8}{|c|}{ Cell number anti-CD19 CAR-T cells } \\
\hline 0 (control) & l) $27.48 \pm 1.92(3 / 3)^{a}$ & $28.26 \pm 1.84(3 / 3)^{\mathrm{a}}$ & $27.94 \pm 1.88(3 / 3)^{\mathrm{a}}$ & $27.14 \pm 2.42(3 / 3)^{\mathrm{a}}$ & $27.36 \pm 2.51(3 / 3)^{\mathrm{a}}$ & $25.8 \pm 0(1 / 3)^{\mathrm{a}}$ & $N A(0 / 3)^{a}$ \\
\hline $1 \times 10^{6}$ & $28.08 \pm 1.42(6 / 6)^{a}$ & $29.07 \pm 1.13(6 / 6)^{\mathrm{a}}$ & $28.97 \pm 1.18(6 / 6)^{a}$ & $28.62 \pm 0.98(6 / 6)^{a}$ & $28.72 \pm 0.94(6 / 6)^{a}$ & $27.57 \pm 0.54(3 / 6)^{\mathrm{a}}$ & $23.05 \pm 0.25(2 / 6)^{a}$ \\
\hline $1 \times 10^{7}$ & $27.95 \pm 1.69(6 / 6)^{a}$ & $27.93 \pm 1.56(6 / 6)^{\mathrm{a}}$ & $27.52 \pm 1.41(6 / 6)^{\mathrm{a}}$ & $26.85 \pm 1.43(6 / 6)^{\mathrm{a}}$ & $26.57 \pm 1.93(6 / 6)^{a}$ & $26.58 \pm 1.78(6 / 6)^{\mathrm{a}}$ & $26.72 \pm 2.36(6 / 6)^{a}$ \\
\hline
\end{tabular}

${ }^{\mathrm{a}}$, number of animals surviving at each time point/number initially in group. CAR, chimeric antigen receptor.

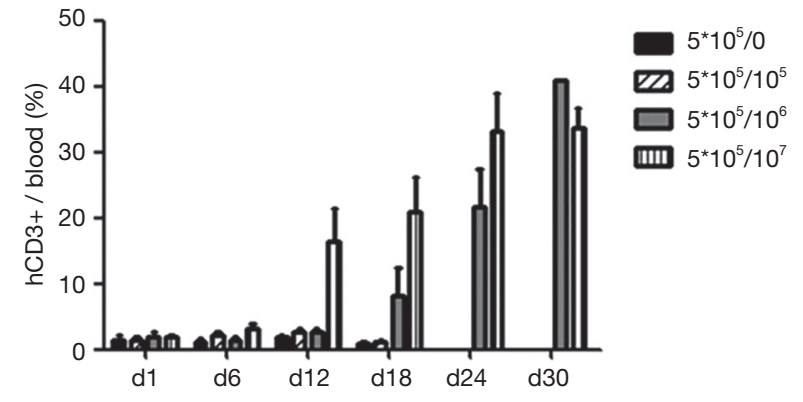

Figure 6 Persistence and proliferation of anti-CD19 CAR T cells in Raji xenograft model. hCD3, human CD3; CAR, chimeric antigen receptor.

a broad range of human organs, was used in functional cross-reactivity studies. As shown in Figure $7 B$, on-target reactivity was observed for anti-CD19 CAR-T cells against K562/CD19+ and Raji cells, which are high CD19-positive $(\geq 97 \%)$. No off-target cross-reactivity was observed for any of the tested cell lines. A slight loss of specificity was observed in the other tested cell lines at a high ET ratio; however, the ET ration was absent in clinical condition.

\section{Tumorigenic potential of anti-CD19 CAR-T cells}

All mice injected with Hela and Raji cells were sacrificed for humane reasons at 3 and 7 weeks post-inoculation. All animals receiving a DBPS survived to the end of study. Survivals of animals receiving anti-CD19 CAR-T cells were shown in Figure 8A. The mice receiving anti-CD19 CAR-T cells exhibited no tumor formation at terminal sacrifice or on the date of death caused by graft-versus-host-disease (GvHD). All animal body weights were monitored prior to, throughout the study, and at sacrifice. Body weights were measured at designated time points and summarized in Table 4. All mice receiving anti-CD19 CAR-T cells appeared clinically normal and body weight changes were within the normal physiological range for 8 weeks. Mice injected with $1 \times 10^{7}$ of anti-CD19 CAR-T cells were observed with a decrease in body weight due to GvHD characterized with ruffled fur, reduced mobility, rashes and weight loss (Figure 8B).

\section{Discussion}

Pre-clinical safety and efficacy studies are essential for translating adoptive $\mathrm{T}$ cell therapy to clinical use. In the present study, a newly generated construct of anti-CD19 CAR-T cells and tested its effectiveness against CD19+ malignancies. We demonstrated that the anti-CD19 CAR-T cells had favorable affinity to CD19 and effectively lysed CD19+ tumor cells in vitro. The anti-CD19 CAR-T cells efficiently eradicated engrafted CD19+ tumors in immunodeficient mice. Our results indicated that the novel construct exhibited long persistence in presence of tumor. We also showed that anti-CD19 CAR-T cells had neither tumorigenic potential in NGS mice nor cross-reactivity to human non-CD19 expressing cells.

Immunotherapy using adoptive transfer of anti-CD19 CAR- $T$ against the $B$ lineage hematological malignancies has achieved favorable success in clinical settings. Therapeutic efficacy of CAR-T cells has correlated with several factors including the duration of their persistence in the circulation (3-9,11-14). Costimulation using costimulatory molecules such as CD28 and 4-1BB has been shown to achieve favorable $\mathrm{T}$ cell activation and longevity of responses. Among these molecules, 4-1BB has been suggested to preferentially enhance the survival CAR-T 
A
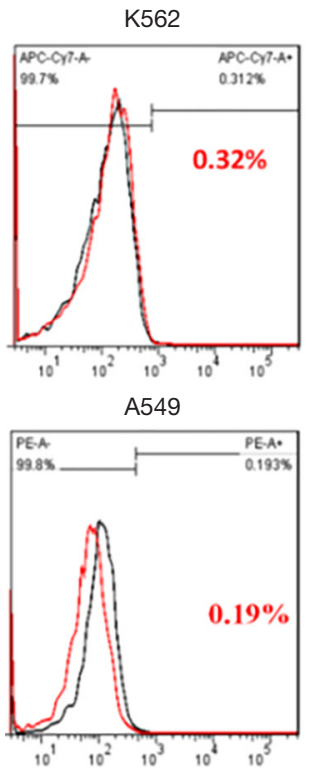

786-O

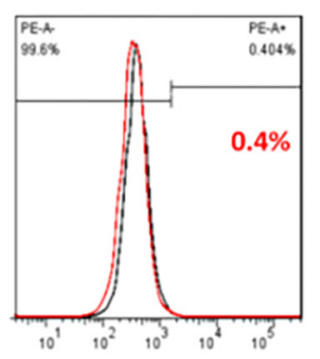

K562/CD19

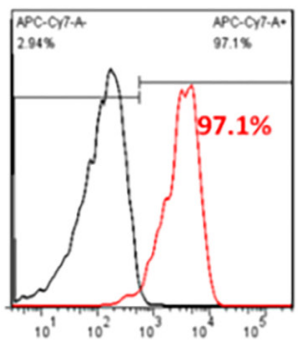

HT-29

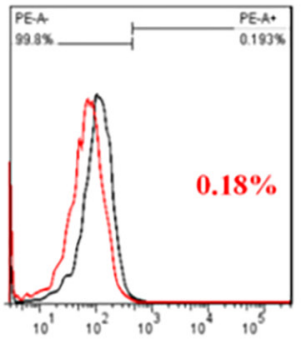

HepG2

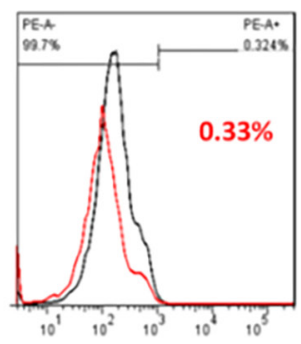

Raji

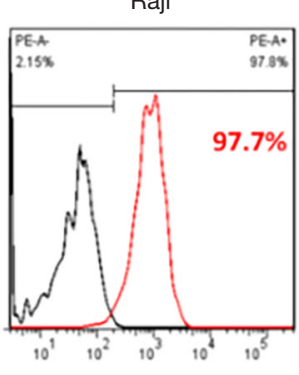

HK2

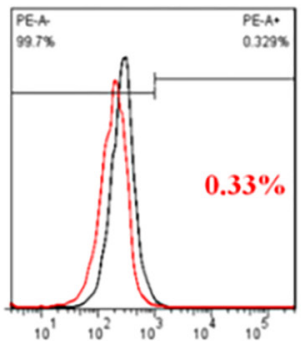

MDA-MB-231

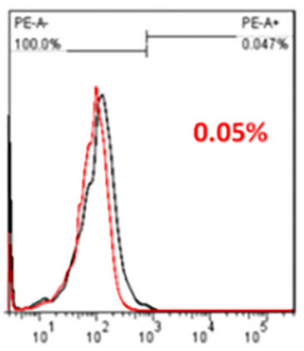

B

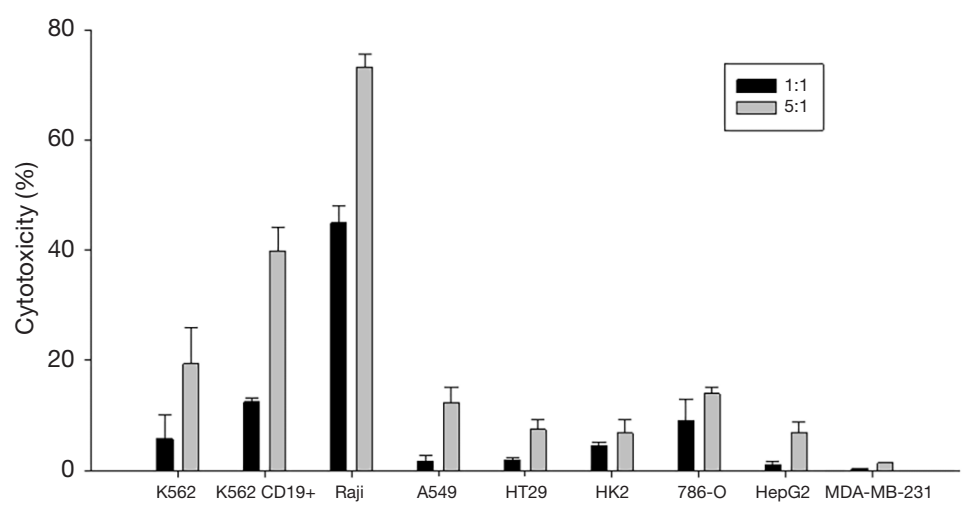

Figure 7 In vitro Cross reactivity of anti-CD19 CAR T cells. (A) Positive rate of CD19 in different target cell lines; (B) anti-CD19 CAR T cells cross-reactivity (off-target reactivity) against human cell lines. CAR, chimeric antigen receptor.

cells, whereas CAR-T cells with CD28 exhibit a rapid cell proliferation $(15,16)$. In addition to costimulation, the use of humanized or human scFV has been suggested to contribute to prolonged persistence of CAR-T cells as a result of reduced immunogenicity to the CAR construct.
In the present study, we developed an anti-CD19 CAR construct incorporating with 4-1BB with an intention to achieve favorable anti-tumor activity. We observed that the anti-CD19 CAR-T cells exerted relatively high specific affinity to CD19 targets and favorable in vitro killing 

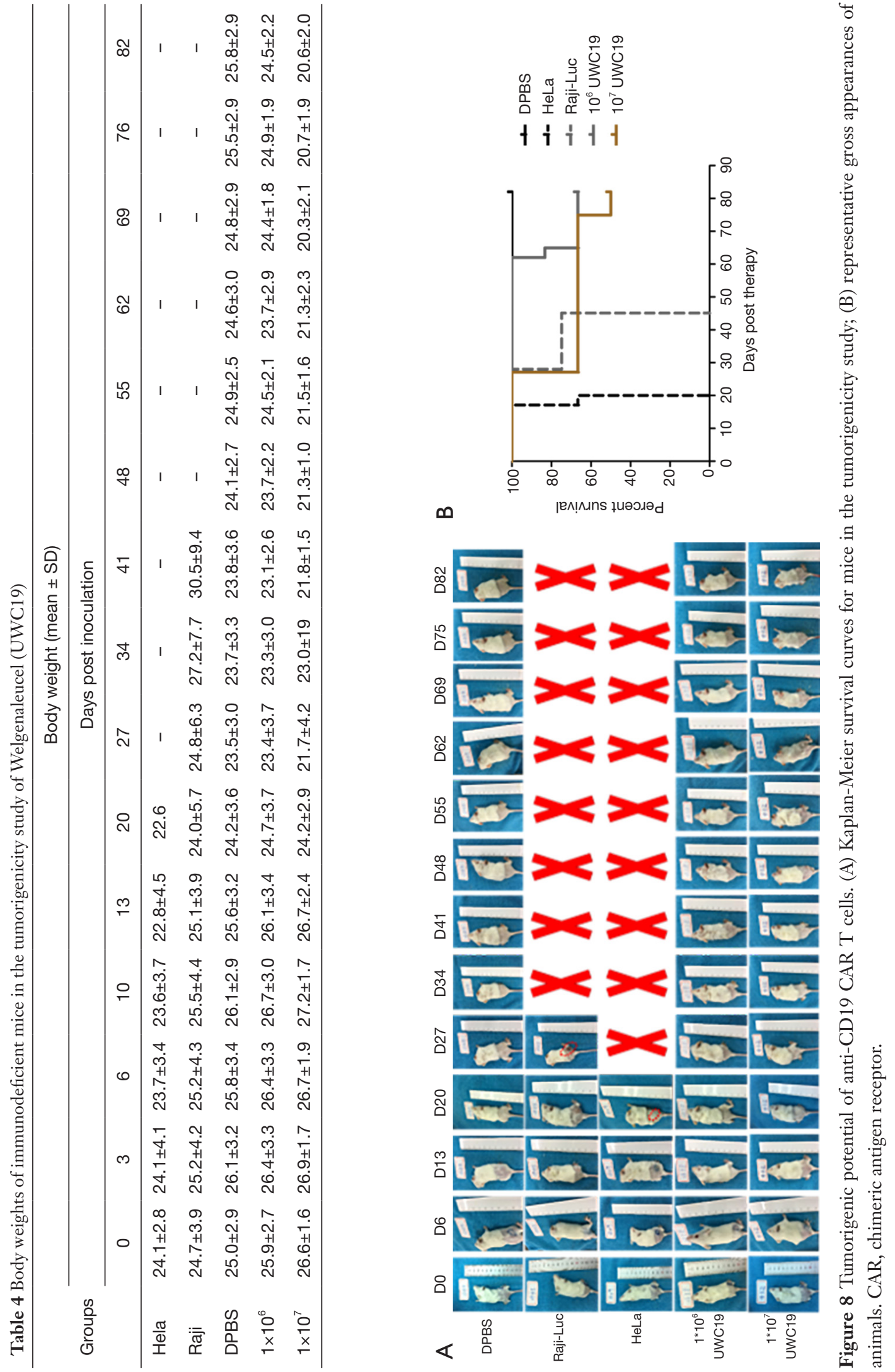
ability against lymphoma and leukemia cells. In addition, 4-1BB moiety coupled in the CAR constructs is postulated to synergistically contribute to the in vitro anti-cancer activities, which is shown to enhance the in vitro function of CARs (17-19). Using a xenograft mouse model, we found that the anti-CD19 CAR-T cells were potent in regressing CD19+ lymphoma xenografts and persisted for 30 days in tumor-bearing immunodeficient mice. However, further non-clinical and clinical studies are necessary to determine the fate and persistence of the anti-CD19 CAR-T cells.

In the tumorigenicity study, the anti-CD19 CAR-T cells caused no tumor formation in 14 weeks after implantation in immunodeficient mice. Two human cell lines, Raji and Hela, used as positive references resulted in significant tumor growth and consequent death of mice. Data on the incidence of spontaneous tumors are not available for immunodeficient mice but are available for other mouse strains $(20,21)$. As expected, GvHD was observed in immunodeficient mice receiving anti-CD19 CAR-T cells. In addition to tumor formation, off-target toxicity occurring when CAR-T cells unspecifically and unexpectedly attack an antigen other than the designated tumor-associated antigen. Off-target toxicity has been reported in patients infused with genetically-modified autologous $\mathrm{T}$ cells expressing an enhanced affinity $\mathrm{T}$-cell receptor (TCR) against MAGE-A3 in testis (22). Offtarget recognition of anti-CD19 CAR-T cells is critical for developments. Due to lack of suitable ex vivo model, we examined the cross reactivity of anti-CD19 CAR-T cells by incubating CAR-T cells with the cells from tissues. Our findings showed that anti-CD19 CAR-T cells specifically recognized CD19+ cells and exerted none toxicity to the other non-CD19 expressing cells, suggesting that the CAR-T cells have relatively low off-target toxicity.

\section{Conclusions}

We develop and characterize an anti-CD19 CAR for it antitumor activity. The CARs are able to reprogram T-cells against a designated target. The CAR-T cells represent an ideal approach to eradicate tumor without tumorigenic potential and off-target toxicity. Further studies are required to determine the dosage of CAR-T cells and to explore potential application in a clinical setting.

\section{Acknowledgments}

Funding: None.

\section{Footnote}

Conflicts of Interest: All authors have completed the ICMJE uniform disclosure form (available at http://dx.doi. org/10.21037/atm.2020.02.148). The authors have no conflicts of interest to declare.

Etbical Statement: The authors are accountable for all aspects of the work in ensuring that questions related to the accuracy or integrity of any part of the work are appropriately investigated and resolved. The experimental protocol was approved by the Taipei Medical University Institutional Animal Care and Use Committee (IACUC) (IACUC No. LAC-2017-0124).

Open Access Statement: This is an Open Access article distributed in accordance with the Creative Commons Attribution-NonCommercial-NoDerivs 4.0 International License (CC BY-NC-ND 4.0), which permits the noncommercial replication and distribution of the article with the strict proviso that no changes or edits are made and the original work is properly cited (including links to both the formal publication through the relevant DOI and the license). See: https://creativecommons.org/licenses/by-nc-nd/4.0/.

\section{References}

1. Curran KJ, Brentjens RJ. Chimeric antigen receptor T cells for cancer immunotherapy. J Clin Oncol 2015;33:1703-6.

2. Maus MV, Grupp SA, Porter DL, et al. Antibodymodified T cells: CARs take the front seat for hematologic malignancies. Blood 2014;123:2625-35.

3. Brentjens RJ, Davila ML, Riviere I, et al. CD19-targeted $T$ cells rapidly induce molecular remissions in adults with chemotherapy-refractory acute lymphoblastic leukemia. Sci Transl Med 2013;5:177ra38.

4. Grupp SA, Kalos M, Barrett D, et al. Chimeric antigen receptor-modified $\mathrm{T}$ cells for acute lymphoid leukemia. $\mathrm{N}$ Engl J Med 2013;368:1509-18.

5. Maude SL, Frey N, Shaw PA, et al. Chimeric antigen receptor $\mathrm{T}$ cells for sustained remissions in leukemia. $\mathrm{N}$ Engl J Med 2014;371:1507-17.

6. Kochenderfer JN, Dudley ME, Kassim SH, et al. Chemotherapy-refractory diffuse large B-cell lymphoma and indolent B-cell malignancies can be effectively treated with autologous $T$ cells expressing an anti-CD19 chimeric antigen receptor. J Clin Oncol 2015;33:540-9. 
7. Porter DL, Hwang W'T, Frey NV, et al. Chimeric antigen receptor $\mathrm{T}$ cells persist and induce sustained remissions in relapsed refractory chronic lymphocytic leukemia. Sci Transl Med 2015;7:303ra139.

8. Brentjens RJ, Riviere I, Park JH, et al. Safety and persistence of adoptively transferred autologous CD19targeted $\mathrm{T}$ cells in patients with relapsed or chemotherapy refractory B-cell leukemias. Blood 2011;118:4817-28.

9. Davila ML, Riviere I, Wang X, et al. Efficacy and toxicity management of 19-28z CAR T cell therapy in B cell acute lymphoblastic leukemia. Sci Transl Med 2014;6:224ra25.

10. Lamers CH, Willemsen R, van Elzakker P, et al. Immune responses to transgene and retroviral vector in patients treated with ex vivo-engineered T cells. Blood 2011;117:72-82.

11. Porter DL, Levine BL, Kalos M, et al. Chimeric antigen receptor-modified $\mathrm{T}$ cells in chronic lymphoid leukemia. N Engl J Med 2011;365:725-33.

12. Kalos M, Levine BL, Porter DL, et al. T cells with chimeric antigen receptors have potent antitumor effects and can establish memory in patients with advanced leukemia. Sci Transl Med 2011;3:95ra73.

13. Lee DW, Kochenderfer JN, Stetler-Stevenson M, et al. T cells expressing CD19 chimeric antigen receptors for acute lymphoblastic leukaemia in children and young adults: a phase 1 dose-escalation trial. Lancet 2015;385:517-28.

14. Turtle CJ, Hanafi LA, Berger C, et al. CD19 CAR-T cells of defined CD4+:CD8+ composition in adult B cell ALL patients. J Clin Invest 2016;126:2123-38.

15. Kawalekar OU, O'Connor RS, Fraietta JA, et al. Distinct

Cite this article as: $\mathrm{Hu} \mathrm{SI}, \mathrm{Ko} \mathrm{MC}$, Dai YH, Lin HA, Chen LC, Huang KY, Pang TL, Kuo CY, Lin HC. Pre-clinical assessment of chimeric antigen receptor $\mathrm{t}$ cell therapy targeting CD19+ B cell malignancy. Ann Transl Med 2020;8(9):584. doi: 10.21037/atm.2020.02.148
Signaling of Coreceptors Regulates Specific Metabolism Pathways and Impacts Memory Development in CAR T Cells. Immunity 2016;44:380-90.

16. van der Stegen SJ, Hamieh M, Sadelain M. The pharmacology of second-generation chimeric antigen receptors. Nat Rev Drug Discov 2015;14:499-509.

17. Imai C, Mihara K, Andreansky M, et al. Chimeric receptors with 4-1BB signaling capacity provoke potent cytotoxicity against acute lymphoblastic leukemia. Leukemia 2004;18:676-84.

18. Wang J, Jensen M, Lin Y, et al. Optimizing adoptive polyclonal $\mathrm{T}$ cell immunotherapy of lymphomas, using a chimeric $\mathrm{T}$ cell receptor possessing CD28 and CD137 costimulatory domains. Hum Gene Ther 2007;18:712-25.

19. Kochenderfer JN, Feldman SA, Zhao Y, et al. Construction and preclinical evaluation of an anti-CD19 chimeric antigen receptor. J Immunother 2009;32:689-702.

20. Krupke DM, Begley DA, Sundberg JP, et al. The Mouse Tumor Biology Database: A Comprehensive Resource for Mouse Models of Human Cancer. Cancer Res 2017;77:e67-e70.

21. Begley DA, Sundberg JP, Krupke DM, et al. Finding mouse models of human lymphomas and leukemia's using the Jackson laboratory mouse tumor biology database. Exp Mol Pathol 2015;99:533-6.

22. Cameron BJ, Gerry AB, Dukes J, et al. Identification of a Titin-derived HLA-A1-presented peptide as a crossreactive target for engineered MAGE A3-directed T cells. Sci Transl Med 2013;5:197ra103. 